Arq. Bras. Med. Vet. Zootec., v.62, n.1, p.47-53, 2010

\title{
Escala de coma de Glasgow pediátrica modificada para cães
}

\author{
[Pediatric Glasgow coma scale modified for dogs] \\ M.B. Andrade, E.F. Cole, J. Evêncio Neto, A.C.J. Silva, G.A.S. Aleixo, A.L.T. Cunha \\ Universidade Federal Rural de Pernambuco \\ 52171-900 - Recife, PE \\ RESUMO
}

\begin{abstract}
Com o objetivo de modificar a escala de coma de Glasgow pediátrica para utilização na medicina veterinária, foram utilizados 30 cães adultos com alterações neurológicas passíveis de avaliação da consciência. A escala modificada para cães foi aplicada em três momentos com intervalos de 48 horas entre eles, resultando em 90 eventos diversos. A escala foi aplicada para avaliação da abertura ocular (AO), da melhor resposta associada à vocalização (MRV) e da melhor resposta motora (MRM). Com a análise fatorial para os indicadores AO, MRV e MRM, obtiveram-se valores iniciais de 2,482, 0,302 e 0,215 , respectivamente. A variância foi de $82,7 \%, 10,1 \%$ e $7,2 \%$, respectivamente, e cumulativa de $82,7 \%, 92,8 \%$ e $100 \%$, respectivamente. A extração esperada do principal fator, AO, foi de 2,482 com variância de $82,7 \%$ e cumulativa de $82,7 \%$. O resultado da avaliação da consciência dos cães foi normal em $10 \%$ dos animais, alteração leve em $20 \%$, moderada em $45 \%$ e grave em $25 \%$, com escores de Glasgow iguais a 15, entre 13 e 14, entre 9 e 12 e entre 8 e 3, respectivamente. A escala de coma de Glasgow pediátrica modificada para cães é ferramenta segura para avaliação da consciência de cães adultos.
\end{abstract}

Palavras-chave: cão, consciência, alteração neurológica

\begin{abstract}
The pediatric Glasgow coma scale was modified for use in veterinary medicine. Thirty adult dogs with neurological alterations susceptible to evaluation of the conscience were studied. The modified scale for dogs was applied at three moments with intervals of 48 hours, resulting in 90 events. The scale was applied for evaluation of the ocular opening (OO), the best answer associated to vocalization (BAV), and the best motor answer (BMA). With the factorial analysis for the indicators $O O, B A V$, and BMA the initial values of 2.482, 0.302, and 0.215, respectively, were obtained. The variance was of $82.7 \%, 10.1 \%$, and $7.2 \%$, respectively, and cumulative of $82.7 \%, 92.8 \%$, and $100 \%$, respectively. The expected extraction of the main factor, OO, was 2.482 with variance of $82.7 \%$ and cumulative of $82.7 \%$. The evaluation result of the conscience was normal in 10\% of the animals, light alteration in 20\%, moderate in $45 \%$, and severe in 25\%, with scores of Glasgow equal to 15, from 13 to 14, nine to 12, and eight to three, respectively. The pediatric Glasgow coma scale modified for dogs was a safe tool for evaluation of the conscience of adult dogs.
\end{abstract}

Keywords: dog, conscience, neurological alteration

\section{INTRODUÇÃO}

O nível de consciência é o grau de alerta comportamental que o indivíduo apresenta. A possibilidade de variação desse parâmetro em pacientes neurológicos torna relevante sua avaliação e mensuração (Simpson e Reilly, 1982; Koizumi, 1998; Teasdale e Murray, 2000).

$\mathrm{Na}$ medicina humana, foram desenvolvidas escalas baseadas na análise do nível de consciência do paciente, atribuindo um escore de

Recebido em 25 de setembro de 2009

Aceito em 9 de fevereiro de 2010

E-mail: moacirvet@hotmail.com 
pontuação que propicie uma ideia global da condição da consciência do paciente, momento a momento (Jouvet, 1969; Simpson et al., 1991; Holmes et al., 2005). Entre as limitações do processo de avaliação, está a discordância no escore de um mesmo paciente quando feito por profissionais diferentes (Raimondi e Hirschauer, 1984) e o fato de os valores de cada item da escala não refletirem, de forma fidedigna, todas as situações relativas à alteração na função da consciência (Seshia et al., 1983). Há, portanto, indicação diferenciada para o uso de escalas, a qual deve ser cuidadosamente analisada (Gill et al., 2006).

Em 1970, foi desenvolvida por Teasdale e Jennett, da Universidade de Glasgow, Escócia, a escala de coma de Glasgow (Teasdale e Jennett, 1974), elaborada para propor consistente avaliação clínica do nível de consciência dos pacientes com dano cerebral. Essa escala é frequente e observada mundialmente na rotina neurológica humana (Koizumi, 1990; Jennett, 1997; Gill et al., 2006). Sua aplicação é rápida, de fácil compreensão e permite concordância entre avaliadores. Por isso, ela tem sido usada rotineiramente, principalmente nos quadros neurológicos agudos e de trauma. Nessa escala são avaliados dois parâmetros importantes: perceptividade relacionada à função cortical e reatividade que diz respeito à função da formação reticular ativadora ascendente - FRAA (Teasdale e Jennett, 1974).

Simpson e Reilly (1982) publicaram a escala de coma de Glasgow pediátrica, pois crianças até dois anos manifestam resposta verbal limitada. Essa escala também obedece à mesma escala ordinal de escores de pontuação descrita na Escala de Coma de Glasgow. Os achados da escala de coma formam a base da tomada de decisão clínica, como a necessidade de atitudes mais precisas como a tomografia computadorizada, intervenção cirúrgica e/ou utilização de fármacos (Teasdale e Jennett, 1974; Teasdale, 1982).

$\mathrm{Na}$ medicina veterinária, houve grandes avanços na neurologia nos últimos anos, porém deve-se ressaltar a necessidade de realização de pesquisas que viabilizem instrumentos e procedimentos clínicos mais seguros e eficazes de pacientes. No intuito de proporcionar um instrumento próprio que atenda aos rigorosos critérios associados à confiabilidade, fácil aplicação, interpretação e padronização da linguagem, este estudo teve o objetivo de modificar a escala de coma de Glasgow pediátrica para uma escala de coma de Glasgow pediátrica modificada para cães, a fim de avaliar e mensurar a consciência de pacientes caninos.

\section{MATERIAL E MÉTODOS}

A pesquisa foi realizada no Serviço de Neurologia Clínica e Psicobiologia (SNCP) do Hospital Veterinário do Departamento de Medicina Veterinária da Universidade Federal Rural de Pernambuco, entre os meses de setembro e dezembro de 2007, após a verificação prévia dos prontuários e autorização dos proprietários. Foram elaboradas fichas clínicas, contendo dados sobre o proprietário e o paciente, e fichas de aplicação da escala de coma de Glasgow pediátrica, que foi adaptada para cães, contendo os indicadores, os tipos de respostas e os respectivos escores. Os indicadores e os escores não foram alterados, contudo foi acrescido um critério, "estímulo verbal/comando/ao grito" ao indicador abertura ocular.

Foram utilizados 30 cães adultos com alterações neurológicas, sem distinção de sexo, raça e peso, provenientes da rotina ambulatorial. Após o preenchimento da ficha clínica, foi procedida a aplicação da escala em três momentos: $\mathrm{M}_{0}-$ dia do primeiro atendimento, $\mathrm{M}_{1}-$ do segundo atendimento e $\mathrm{M}_{2}$ - do terceiro atendimento, com intervalos de 48 horas cada, totalizando 90 análises.

A escala foi aplicada para análise de três indicadores - abertura ocular (AO), melhor resposta associada à vocalização (MRV) e melhor resposta motora (MRM), havendo pequenas alterações de terminologia para se adequar à veterinária. Cada um dos indicadores foi analisado pelos respectivos critérios, atribuindo-se uma pontuação quando o critério em questão era positivo (Tab. 1). Finalizada a aplicação da escala, foi procedido o somatório dos escores, obtendo um valor total correspondente ao nível de consciência do paciente. 
Tabela 1. Escala de coma de Glasgow pediátrica modificada para cães

\begin{tabular}{llc}
\multicolumn{1}{c}{ Indicador } & \multicolumn{1}{c}{ Critério/resposta } & Escore \\
\hline Abertura ocular & Espontânea & 4 \\
& Estímulo verbal/comando & 3 \\
& Estímulo verbal/comando/ao grito & 3 \\
& Estímulo doloroso & 2 \\
& Sem abertura & 1 \\
Melhor resposta à vocalização & Latido/rosnado & 5 \\
& Choramingo irritado & 4 \\
& Choramingo à dor & 3 \\
& Ganido à dor & 2 \\
Melhor resposta motora & Sem resposta & 1 \\
& Movimento espontâneo e normal & 6 \\
& Reação ao toque & 5 \\
& Reação à dor & 4 \\
Total & Flexão anormal - descorticação & 3 \\
& Extensão anormal - descerebração & 2 \\
& Nenhuma & 1 \\
\hline
\end{tabular}

Ao aplicar a escala de coma de Glasgow, atribuise um escore numérico a cada um dos indicadores e calcula-se a pontuação total. O escore total mínimo é 3, revelando arreatividade, e o máximo é 15 , mostrando que o doente está desperto, alerta e totalmente responsivo. Quanto à classificação associada à gravidade, considerase alteração grave o intervalo entre 3-8, moderada entre 9-12, leve entre 13-14 e normal 15. A queda de três escores na escala foi considerada como sinal de alerta, pois o paciente poderia estar mudando de faixa na classificação de gravidade (Teasdale e Jennett, 1974; Teasdale, 1982; Teasdale e Murray, 2000).

Os critérios dois e três foram modificados e se apresentam com nomenclatura diferenciada da escala de coma de Glasgow pediátrica e subdivididos mediante entonação da voz do examinador, adequando-se melhor aos animais. $\mathrm{Na}$ escala de coma de Glasgow pediátrica, a pontuação estabelecida para os critérios 1 a 4 é de 04 a 01, respectivamente. Na escala de coma de Glasgow pediátrica modificada para cães, apesar de existirem cinco critérios, a pontuação é de 04 a 01 , pois os critérios dois e três possuem o mesmo escore.

Após a obtenção dos dados, procedeu-se ao tratamento estatístico por meio de análise fatorial, um dos exames mais utilizados na validação de métodos de aplicação de testes (Mardia et al., 1979; Hair et al., 2005). O programa utilizado foi o Statistical Package for the Social Sciences (Altman, 1991; Zar, 1999).

Nas amostras deste trabalho, foram utilizados escores ou nota média ponderada, para representar um conjunto com maior dimensão em um cômputo com dimensão única. Por exemplo, se um elemento de $\mathbf{y}$ tem distribuição normal padrão, a probabilidade de esse elemento, digamos $y_{i}$, ser maior que 1,28, por exemplo, é de aproximadamente $90 \%$. Isto quer dizer que cerca de $90 \%$ dos elementos de uma população com distribuição normal tem valores acima de 1,28 .

\section{RESULTADOS E DISCUSSÃO}

A resposta associada à vocalização, quando presente de forma coerente, indica o mais alto grau de integração do sistema nervoso central. Os cães não verbalizam com linguagem, mas com sons específicos. Dessa maneira, a escala de coma de Glasgow pediátrica modificada para cães comprova as afirmações de Simpson e Reilly (1982) quanto à necessidade de uma alteração da escala de coma de Glasgow, no sentido de avaliar o nível de consciência em pacientes que não falam, visando à enunciação do pensamento, utilizando-se, assim, a escala de coma de Glasgow pediátrica.

$\mathrm{Na}$ escala de coma de Glasgow pediátrica, o indicador MRV apresenta cinco critérios, 1- 
balbucia, 2- choro irritado, 3- choro à dor, 4gemido à dor e 5- sem resposta (Simpson e Reilly, 1982). Na escala de coma de Glasgow pediátrica modificada para cães, os critérios de um a cinco foram denominados, respectivamente, de latido/rosnado, choramingo irritado, choramingo à dor, ganido à dor e ausência de resposta. A pontuação preconizada na escala de coma de Glasgow pediátrica para os critérios um a cinco é de 05 a 01, respectivamente, sendo a mesma estabelecida na escala de coma de Glasgow pediátrica modificada para cães.

$\mathrm{Na}$ escala de coma de Glasgow pediátrica modificada para cães, o indicador MRM não sofreu alterações, constando dos mesmos critérios e pontuações apresentadas na escala de coma de Glasgow pediátrica, pois a terminologia utilizada é adequada à medicina veterinária.

A escala de coma de Glasgow pediátrica modificada para cães obedeceu à mesma escala ordinal de escores de pontuação descrita na escala de coma de Glasgow e na escala de coma de Glasgow pediátrica (Pereira et al., 1999). Dessa forma, na presença de um escore menor ou igual a oito, houve necessidade de intubação traqueal e hiperventilação, o que também foi observado por Durhan et al. (2000).

O número de animais utilizados neste experimento foi satisfatório para análise fatorial, pois as 90 observações foram consideradas independentes, pois esses animais foram submetidos a três aplicações do protocolo da escala de coma de Glasgow pediátrica modificada para cães, em momentos distintos, considerando-se, dessa forma, uma avaliação independente, estreitamente associada à evolução clínica de cada paciente, visto que, de acordo com Hair et al. (2005), o número mínimo da amostra é cinco vezes o número de variáveis (a proporção neste trabalho foi de 30 vezes).

A análise fatorial é um dos exames estatísticos mais utilizados na validação de métodos de aplicação de testes, podendo ser utilizado para examinar padrões ou variáveis latentes, considerando as informações de um grande conjunto de variáveis em um conjunto de menor número. A análise fatorial define uma estrutura subjacente em uma matriz de dados, abordando a correlação entre um grande conjunto de variáveis, como é o caso dos escores (Mardia et al., 1979; Hair et al., 2005).

O número de animais sadios atendidos pelo SNCP é de $10 \%$, o que representaria o valor de 1,28 na tábua da distribuição normal. Em estado leve é de $20 \%$, o que na tábua da normal equivale ao valor de 0,52 , pois a probabilidade de $\mathrm{y}_{\mathrm{i}}$ exceder 0,52 é de $30 \%(10 \%+20 \%)$; em estado moderado é de $45 \%$, o que equivale ao valor $-0,62$, pois a probabilidade de $\mathrm{y}_{\mathrm{i}}$ exceder esse valor é de $75 \%(10 \%+20 \%+45 \%)$; e, finalmente, os $25 \%$ restantes estariam abaixo de 0,67 , significando o estado grave.

Segundo Mardia et al. (1979) e Hair et al. (2005), a análise fatorial pode gerar até três fatores, mas, pela Tab. 2, apenas o primeiro é responsável por cerca de $80 \%$ da variabilidade total, sendo fortemente recomendada sua aplicação. Esse fator é compreendido na aplicação da escala de coma de Glasgow pediátrica modificada para cães como AO, MRV e RMR.

Tabela 2. Análise fatorial dos indicadores de variância total por meio da análise do principal fator obtida em cães

\begin{tabular}{ccccccc}
\hline & \multicolumn{3}{c}{ Valores iniciais } & \multicolumn{2}{c}{ Extração esperada } \\
\hline Fator & Total & Variância, \% & Cumulativa, \% & Total & Variância, \% & Cumulativa, \% \\
AO & 2,482 & 82,743 & 82,743 & 2,482 & 82,743 & 82,743 \\
MRV & 0,302 & 10,080 & 92,823 & & & \\
MRM & 0,215 & 7,177 & 100,000 & & & \\
\hline
\end{tabular}

AO: abertura ocular; MRV: melhor resposta à vocalização e MRM: melhor resposta motora.

A Fig. 1 refere-se ao Q-Q Plot que representa uma ferramenta gráfica que relaciona o quantil da variável observada e o quantil de uma distribuição, em que Q-Q indica Quantil-Quantil, que é útil para verificar se os dados, ou a sua transformação, se aproximam de uma distribuição específica, neste caso, a distribuição normal. Ainda na Fig. 1, demonstram-se os valores associados a uma série de tabelas, onde se encontram as explicações para a construção 
dos fatores, e indicam que a distribuição normal dos escores pode ser adotada. Os pontos são os valores dos escores calculados pela equação de análise fatorial que usa o primeiro fator. No eixo $\mathrm{X}$, estão os valores dos escores obtidos e, no eixo $\mathrm{Y}$, os valores que cada escore representa na distribuição normal. Se eles fossem os mesmos (valores da normal e valores estimados), todos os pontos estariam sobre a reta. Quanto mais próximo os pontos estiverem da linha, melhor para supor normalidade (o ideal seria que todos os pontos estivessem sobre ela).

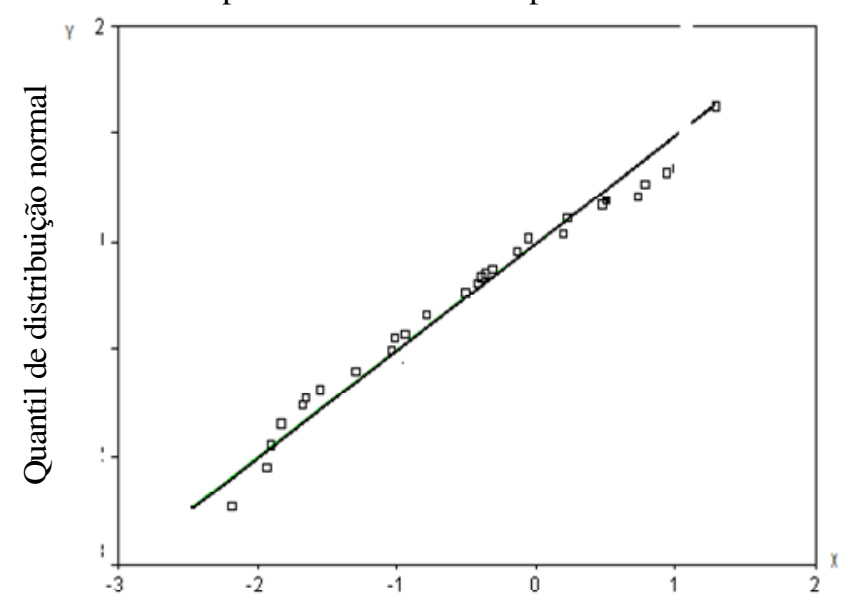

Quantil dos valores dos escores observados em cães

Figura 1. Distribuição dos valores dos escores obtidos pela análise fatorial em relação à distribuição normal em cães.

A Fig. 2 refere-se ao gráfico dos resíduos (diferença entre os valores observados e os valores produzidos pela análise fatorial) em relação aos valores observados e auxilia a verificação de independência dos mesmos.
Ambos evidenciam essa aplicação, demonstrando que os escores obtidos pela análise fatorial se aproximam da distribuição normal, corroborando com Mardia et al. (1979) e Hair et al. (2005).

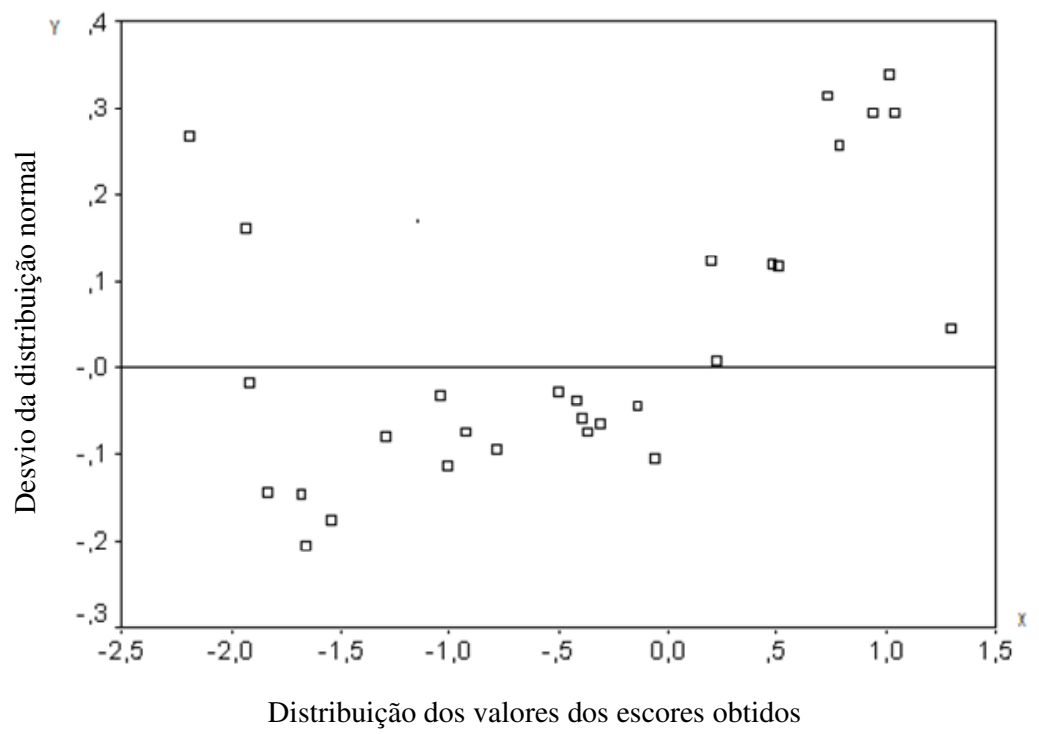

Figura 2. Resíduos (\%) obtidos dos escores pela análise fatorial em relação ao desvio da distribuição normal em cães. 
Estes resultados estão vinculados à Tab. 3 de contingência dois com as três respostas para cada animal. A soma obtida pelas respostas classifica os escores quanto à gravidade em normal, leve, moderada ou grave, conforme o fizeram Teasdale e Jennett (1974), Teasdale (1982) e Teasdale e Murray (2000) que classificaram os níveis de consciência em seus pacientes humanos após a soma dos escores.

Na aplicação da escala de coma de Glasgow pediátrica modificada para cães foi atribuído um escore numérico a cada um dos indicadores permitindo o cálculo de uma pontuação total. $\mathrm{O}$ escore total mínimo foi três, quando se observou o estado de coma, e o máximo foi 15 , que evidenciando que o paciente estava desperto, alerta e completamente responsivo, conforme afirmaram Teasdale e Jennett (1974), Teasdale (1982) e Teasdale e Murray (2000), acerca dos mesmos parâmetros de aplicação para os seres humanos.

De acordo com Teasdale e Jennett (1974), Teasdale (1982), Teasdale e Murray (2000), a classificação da gravidade é obtida quando a soma dos escores for: 15 para paciente normal, 14-13 para paciente leve, 12-9 para moderada e 8-3 para grave, semelhantes às classificações encontradas na escala de coma de Glasgow pediátrica modificada para cães deste estudo, observada na Tab. 3.

Tabela 3. Classificação dos cães segundo a análise fatorial dos dados obtidos e Glasgow (gravidade e somatório)

\begin{tabular}{lccc}
\multicolumn{1}{c}{ Estado } & $\begin{array}{c}\text { Animais/atendimento } \\
(\%)\end{array}$ & $\begin{array}{c}\text { Escala de Glasgow } \\
\text { (escore) }\end{array}$ & Escala de distribuição normal \\
\hline Normal & 10 & Igual a 15 & Acima de 1,28 \\
Leve & 20 & Entre 13 e 14 & Entre 0,52 e 1,28 \\
Moderado & 45 & Entre 9 e 12 & Entre $-0,67$ e 0,52 \\
Grave & 25 & Entre 8-3 & Abaixo de $-0,67$ \\
\hline
\end{tabular}

No intuito de entender a validade do método, basta tomar como exemplos duas amostras das 90, avaliadas no experimento (Tab. 4). O animal um, por exemplo, obteve respostas 04, 03 e 04 para AO, MRV, MRM, respectivamente; ao aplicar a transformação, esse paciente alcançou o escore 0,21975, permanecendo entre os valores 0,67 e 0,52 , que o classifica como estado moderado. Neste caso o animal obteve soma igual a 11, que o classifica, segundo a escala de coma de Glasgow, também como estado moderado. $\mathrm{O}$ animal dois teve respostas $04,05 \mathrm{e}$ 06, que resultou em escore 1,29288, que o classificou como normal (maior que 1,28), a mesma classificação se tivesse sido feita pela escala de coma de Glasgow (Teasdale e Jennett, 1974; Teasdale, 1982; Teasdale e Murray, 2000).

Tabela 4. Resposta à avaliação experimental em cães

\begin{tabular}{ccccccc}
\hline Amostras & AO & MRV & MRM & Soma & Resultados & Classificação \\
\hline 1 & 4 & 3 & 4 & 11 & 0,21975 & moderada \\
2 & 4 & 5 & 6 & 15 & 1,29288 & normal \\
\hline
\end{tabular}

AO: abertura ocular; MRV: melhor resposta à vocalização; MRM: melhor resposta visual.

A escala de coma de Glasgow pediátrica modificada para cães avaliou e mensurou os níveis de consciência dos cães, com alterações neurológicas presentes nesta pesquisa, o que está de acordo com Jouvet (1969), Teasdale e Jennett (1974), Simpson e Reilly (1982), Pal et al. (1989), Jennett (1997), Koizumi, (1990), Koizumi (1998), Teasdale e Murray (2000) com suas análises em pacientes humanos portadores de alterações neurológicas.
A escala de coma de Glasgow pediátrica modificada para cães poderá ser utilizada por médicos veterinários, enfermeiros veterinários ou pesquisadores, como um instrumento de mensuração da consciência, que avalia o paciente diante de um estímulo quanto à capacidade de abrir os olhos, da melhor resposta à vocalização e da melhor resposta motora, o que confirma as observações obtidas em pacientes humanos (Teasdale e Jennett, 1974; Teasdale, 1982). 


\section{CONCLUSÃO}

Conclui-se que a escala de coma de Glasgow pediátrica modificada para cães é ferramenta segura para avaliação da consciência em cães adultos na rotina ambulatorial, tendo sua validade construída por meio da análise fatorial.

\section{REFERÊNCIAS BIBLIOGRÁFICAS}

ALTMAN, D.G. Practical statistics for medical research. London: Chapman and Hall, 1991. $611 \mathrm{p}$.

DURHAM, S.R.; $\quad$ CLANCY, R.R.; LEUTHARDT, E. et al. Infant coma scale ("infant face scale"): a novel coma scale for children less than two years of age. $J$. Neurotrauma, v.17, p.729-737, 2000.

GILL, M.; STEELE, R.; WINDEMUTH, R. et al. Comparison of five simplified scales to the out-of-hospital glasgow coma scale for the prediction of traumatic brain injury outcomes. Acad. Emerg. Med., v.13, p.968-973, 2006.

HAIR Jr., J.F.; ANDERSON, R.E.; TATHAM, R.L. et al. Análise Multivariada de Dados. 1.ed. Porto Alegre: Bookman, 2005. p.89-110.

HOLMES, J.F.; PALCHAK, M.J.; MacFARLANE, T. Performance of the Pediatric Glasgow Coma Scale in Children with Blunt Head Trauma. Acad. Emerg. Med., v.12, p.814819, 2005.

JENNETT, B. The history of the Glasgow Coma Scale: an interview with professor Bryan Jennett. Interview by Carole Rush. Int. J. Trauma Nurs., v.3, p.114-118, 1997.

JOUVET, M. Coma and other disorders of consciousness. In: VINKEN, P.J.; BRUYN, G.N. (Eds). Handbook of clinical neurology. Amsterdam: North Helland Publishing, 1969. v.3, cap.5, p.62-79.

KOIZUMI, M.S. Método de avaliação do nível de consciência e interpretação. Acta Paul. Enferm., v.3, p.17-24, 1990.
KOIZUMI, M.S. Avaliação do nível de consciência. In: CONGRESSO BRASILEIRO DE NEUROLOGIA, 1998, São Paulo. Anais... São Paulo, Acad. Bras. Neurol., v.4, p.39:1-12, 1998.

MARDIA, K.V.; KENT, J.T.; BIBBY, J.M. Multivariate analysis. 1.ed. London: Academic, 1979. cap.9, p.255-267.

PAL, J.; BROWN, R.; FLEISZER, D. The value of de Glasgow Coma Scale and injury severity score: prediting outcome in multiply trauma patients with head injury. J. Trauma - Injury Infec. Crit. Care, v.29, p.746-748, 1989.

PEREIRA Jr., G.A.; ANDREGHETTO, A.C.; BASILE-FILHO, A. et al. Trauma no paciente pediátrico. Medicina, v.32, p.262-281, 1999.

RAIMONDI, A.J.; HIRSCHAUER, J. Head injury in the infant and toddler: coma scoring and outcome scale. Child's Brain, v.11, p.12-35, 1984.

SESHIA, S.S.; JOHNSTON, B.; KASIAN, G. Non-traumatic coma in childhood: critical variables in prediction of outcome. Develop. Med. Child Neur., v.25, p.492-501, 1983.

SIMPSON, D.A.; REILLY, P. Pediatric coma scale. Lancet, v.2, p.450, 1982.

SIMPSON, D.A.; COCKINGTON, R. A.; HANIEH, A. et al. Head injuries in infants and young children: the value of the Pediatric coma scale. Childs Nerv. Syst., v.7, p.183-190, 1991.

TEASDALE, G.; JENNETT, B. Assessment of coma and impaired consciousness. A practical scale. Lancet, v.13, p.81-84, 1974.

TEASDALE, G. The description of conscious level: a case for the Glasgow coma scale. Scot. Med. J., v.27, p.7-9, 1982.

TEASDALE, G.M.; MURRAY, L. Revisiting the Glasgow coma scale and coma score. Intens. Care Med., v.26, p.153-154, 2000.

ZAR, J.H. Biostatistical analysis. 4.ed. New Jersey: Prentice Hall, 1999. 929p. 